\title{
Pea Hull Fiber Improves Bowel Regularity in Both a Healthy and Constipated Rat Model
}

\author{
Julie Marcotorchino', Julien Roux¹, Bruno Bariohay¹, Laetitia Guerin-Deremaux², \\ Clémentine Thabuis ${ }^{2 *}$
}

${ }^{1}$ Biomeostasis, Marseille, France

${ }^{2}$ ROQUETTE, Lestrem, France

Email: *clementine.thabuis@roquette.com

How to cite this paper: Marcotorchino, J., Roux, J., Bariohay, B., Guerin-Deremaux, L. and Thabuis, C. (2021) Pea Hull Fiber Improves Bowel Regularity in Both a Healthy and Constipated Rat Model. Food and Nutrition Sciences, 12, 950-961.

https://doi.org/10.4236/fns.2021.1210070

Received: August 9, 2021

Accepted: October 19, 2021

Published: October 22, 2021

Copyright $\odot 2021$ by author(s) and Scientific Research Publishing Inc. This work is licensed under the Creative Commons Attribution International License (CC BY 4.0).

http://creativecommons.org/licenses/by/4.0/

(c) (i) Open Access

\begin{abstract}
Constipation is an area of concern in people's health. Consequently, health care practitioners recommend lifestyle changes including increasing fiber intake and exercise. This study examined the effect of pea hull fiber (PHF) on loperamide-induced constipation in rats. The rats were fed either a vehicle diet or a diet enriched with PHF. The groups were divided into either a saline or loperamide injection to induce sub-acute constipation. Fecal Pellet Output (FPO) was measured for 3 days. In the saline-treated rats, PHF increased wet and dry fecal weights on day 1 and 2, and increased FPO on day 2 compared to the saline-treated rats on the vehicle diet. In the loperamide model, PHF increased FPO, had higher wet and dry fecal weights on day 1 compared to the loperamide-treated vehicle diet rats. Addition of PHF into the diet may help improve intestinal transit in a loperamide-induced constipation rat model.
\end{abstract}

\section{Keywords}

Pea Hulls, Fiber, Constipation, Transit

\section{Introduction}

For many people, bowel irregularity is an area of great concern in their health [1]. Constipation is clinically defined as infrequent and difficult defecation $(<3$ bowel movements per week), feeling of incomplete evacuation, and passage of hard stools in addition to abdominal discomfort and bloating. It has been estimated that $\sim 15 \%$ of adults worldwide suffer from constipation [1]. There are many people, that do not meet the clinical definition of constipation, yet they 
still have abdominal discomfort (including irregular bowel movements, intestinal pain and bloating). It is interesting to note that this type of sub-clinical constipation is more common in females than males and it increases with age [2]. Just as in patients with constipation, if left untreated, these patients may experience a decrease in quality of life, disturbance of everyday activities, and even depression [2]. In response to these concerns, health care practitioners typically recommend dietary and lifestyle modifications including increasing fiber and water intake and increasing physical exercise. Most western countries fall short on the daily recommended intake of whole grain, vegetables, and overall fiber. While the recommended fiber intake is 25 to $35 \mathrm{~g}$ each day, most adults only consume 16 to $19 \mathrm{~g}$ daily [3]. In Canada, it has even been stated that, amazingly, the main source of dietary fiber is white flour [4]!

Recent systematic literature reviews on the role of fiber supplementation in patients with chronic constipation, identified 13 clinical trials and 9 different types of dietary fiber. These reviews found that when consumed in adequate amounts dietary fibers can help improve stool consistency, and stool frequency [5] [6] [7]. Based on previous studies, four characteristics of fiber are critical for clinical efficacy in patients with constipation including solubility, rate of fermentation, viscosity and gel formation [8]. Soluble fibers are fermented into the colon, absorb water and form a gel in the intestine. The ability of soluble fibers to hold water helps soften the stool making it easier to pass. On the other hand, insoluble fibers are not extensively fermented, do not absorb water, but they do provide bulk which is helpful in improving intestinal transit time. Both soluble and insoluble fibers have been well-studied in various therapeutic areas; however, a small number have focused on the management of chronic constipation, and an even smaller number of trials have studied insoluble fiber [9].

Oat hull fiber, an insoluble fiber, is isolated from the outer most layer of the oat grain and is comprised of $\sim 85 \%$ - $90 \%$ of non-digestible carbohydrate, most of it as insoluble fiber (cellulose and hemicellulose). To date three human studies have examined the effect of oat hull fiber supplementation in patients with chronic constipation [10] [11]. Overall, these studies reported a global laxative effect of oat hull fiber in these patients. Interestingly, to our knowledge only one study has evaluated the effect of pea hull fiber, another insoluble fiber, on intestinal transit in rats. In this study by Hansen et al., supplementation of a rat diet with pea hulls (13.5 $\mathrm{g}$ of fibers/100g of dry diet) decreased transit time from $37 \mathrm{~h}$ in the vehicle group to $23 \mathrm{~h}$ in the supplemented group [12]. Given the beneficial clinical effects reported with oat hull fiber and chronic constipation, coupled with the rat study demonstrating improved transit time with pea hull fiber (PHF) we hypothesized that addition of PHF in a loperamide-induced constipation rat model [13] [14] would help improve bowel function. PHF has been chosen for this study compared to other hulls because pea crops are increasing today for vegetal protein production and PHF has become a highly available by-products with potential health benefits to investigate. 


\section{Materials and Methods}

\subsection{Ethical Statement}

All the experiments were conducted according to the French Regulations for Animal Experimentation and authorized under the project number APAFIS\#16287201807251642419 v2.

\subsection{Study Design: Animal Management}

Sixty, male, $150 \mathrm{~g}$ Wistar rats (Janvier Labs, St Berthevin, France) were individually housed in a high performance Individually Ventilated Cages rack (Innorack $^{\oplus}$, INNOVIVE, France). Food and water were ad libitum. All animals were maintained in a temperature $\left(22.0^{\circ} \mathrm{C} \pm 2^{\circ} \mathrm{C}\right)$ and humidity $(40 \%-50 \%)$ controlled room on a 12-h light (07:00 AM-07:00 PM)/12-h dark cycle.

The vehicle diet (containing $4.9 \%$ crude fibers), as well as the diet containing a fiber supplementation with PHF (ROQUETTE, France) (vehicle diet $+11 \%$ pea hulls fiber corresponding to an $8 \%$-fiber enrichment as pea hulls contains $93 \%$ of fiber in the final product) were purchased from SSNIFF and had the same formulation procedure (1534 SM/RM control; SSNIFF, Germany). PHF was composed with $93 \%$ fiber with $50 \%$ as cellulose, $20 \%$ as hemicellulose and $23 \%$ as pectin and was the only product used for the fiber supplementation of the control diet.

During the 11-day acclimation period (Figure 1), all animals were fed the vehicle diet after which they were randomized to either the vehicle diet $(n=30)$ or the PHF-enriched diet $(n=30)$ for 3 days. Beginning on the $6^{\text {th }}$ day of the acclimation period, the animals were habituated to intraperitoneal (ip) injections with daily administration of saline $(10 \mathrm{~mL} / \mathrm{Kg})$ for 6 days. On Day 1 of the experiment, the rats were matched by body weight and divided into four homogenous groups ( $\mathrm{n}=15$ /group) as follows: vehicle diet/saline, vehicle diet/loperamide, pea hull diet/saline, or pea hull diet/loperamide.

The primary objective was to determine the impact of PHF supplementation on fecal pellet output (FPO) in both healthy and constipated rats. The secondary objectives were fecal wet and dry weight. Food consumption and body weight gain were measured to evaluate the pea hulls intake. At the end of the experimental period, the rats were euthanized according to European ethical regulation.

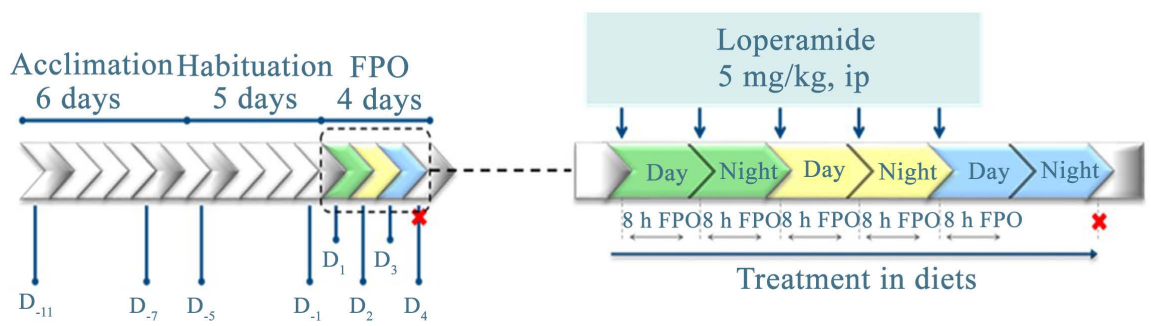

Figure 1. Animal study procedure. 


\subsection{Food intake and Body Weight Measurements}

During the acclimation period described above, body weight and food intake were measured every day in order to ensure an optimal habituation to the injection procedures. On the four days of the experimental procedure itself, the 24 h-food intake and body weight of each animal were measured daily.

\subsection{Loperamide-Induced Constipation}

Loperamide is a $\mu$-opioid receptor agonist widely applied for establishing constipation animal models. Beginning on Day 1 through Day 3 of the experiment, the rats received ip injections of either saline or loperamide hydrochloride at 5 $\mathrm{mg} / \mathrm{kg}$ (Sigma-Aldrich, St Quentin-Fallavier, France) [15] [16]. The rats received ip injections every $12 \mathrm{~h}$ beginning at 8:30 am on Day 1 of the experiment for a total of 5 injections.

\subsection{Treatment}

Beginning on Day 1 of the experiment (08:00 AM) to the end of the study (Day $3)$, rats were fed either with the vehicle diet $(\mathrm{N}=29$ : vehicle/saline $(\mathrm{n}=14)$ and vehicle/loperamide $(\mathrm{n}=15)$ groups) or the pea hulls-enriched $\operatorname{diet}(\mathrm{n}=30$ : pea hulls/saline ( $\mathrm{n}=15)$ and pea hulls/loperamide $(\mathrm{n}=15)$ groups).

Diets were presented in pellet form.

\subsection{FPO Measurements}

On Day 1 (Figure 1), at 8:00 AM, treatment started for four days through the diets. Thirty minutes later (8:30 AM), animals received an ip injection of either saline or loperamide at $5 \mathrm{mg} / \mathrm{kg}$ (ip $\left.{ }^{\circ} 1\right)$. One hour after the ip $n^{\circ} 1$ (9:30 AM), a FPO measurement was performed over 8 hours (diurnal phase 1), including feces counts, frequency, and fecal wet weight. After this first FPO, the feces produced during the following four hours were collected and were withdrawn from the cage preventing coprophagia. At 8:30 pm the animals received a second ip injection of either saline or loperamide (ip $n^{\circ} 2$ ) and FPO measurements were performed over 8 hours during the first night (nocturnal 1), including feces counts, frequency and fecal wet weight. After this second FPO, the feces produced during the following four [4] hours were collected.

On Day 2, at 8:30 am, the animals received a third ip injection of either saline or loperamide at $5 \mathrm{mg} / \mathrm{kg}$ (ip $\mathrm{n}^{\circ} 3$ ). One hour after the ip $\mathrm{n}^{\circ} 3$ (9:30 AM), the third FPO measurement was performed over 8 hours (diurnal 2) including feces counts, frequency and fecal wet weight. After the third FPO measurement, the feces produced during the following four hours were collected. At 8:30 pm, the animals received the fourth ip injection of either saline or loperamide at $5 \mathrm{mg} / \mathrm{kg}$ (ip $n^{\circ} 4$ ). After ip $n^{\circ} 4$, a fourth FPO measurement was performed over 8 hours during the second night (nocturnal 2), including feces counts, frequency and fecal wet weight. After this fourth FPO measurement, the feces produced during the following four hours were collected. 
On Day 3, at 8:30 am, the animals received the last ip injection of either saline or loperamide at $5 \mathrm{mg} / \mathrm{kg}$ (ip ${ }^{\circ} 5$ ). One hour after the ip $\mathrm{n}^{\circ} 5$, a fifth and last FPO measurement was performed over 8 hours (diurnal 3), including feces counts, frequency and fecal wet weight. Consequently, the FPO measurement on Day 3 lasted eight diurnal hours.

FPO was based on the following principle: every 30 minutes over 8 hours, the number of fecal pellets was noted, and the feces were then collected in a single pre-weighed $50 \mathrm{ml}$ falcon (falcon + cap). The Falcon was always hermetically sealed between each feces collection in order to prevent moisture loss. At the end of each FPO measurement, each fraction was weighed (=wet weight). Then, the fecal pellets were dehydrated in a dry heat oven, heated to about $55^{\circ} \mathrm{C}-60^{\circ} \mathrm{C}$ for $48 \mathrm{~h}$ (=dry weight). The percentage of water content was estimated for each fraction by the difference between its wet weight and its dry weight. For each fraction between the FPO measurements ( 4 hours of harvest), the fecal pellets were counted and collected into a single pre-weighed $50 \mathrm{ml}$ falcon (falcon + cap). Then the fecal pellets were dehydrated in a dry heat oven, heated to about $55^{\circ} \mathrm{C}$ $60^{\circ} \mathrm{C}$ for $48 \mathrm{~h}$ in order to obtain the dry weight.

Food intake was also monitored all along FPO measurement.

\subsection{Statistical Analyses}

Data are presented as mean \pm SEM. Statistical analyses were performed with the Statview 5.0.1 program (Statview software, Cary, NC, USA).For the comparison of four groups statistical analyses were performed using a repeated-measures ANOVA for body weight, body weight gain and food intake and a one-way ANOVA for the other parameters (for example: number of fecal pellets). When ANOVA analyses revealed a significant effect, post-hoc analyses were done using a Fischer's PLSD test. Risk a was fixed at 0.05.

\section{Results}

\subsection{Body Weight and Food Intake}

Body weight and body weight gain were not different between the groups during the experimental period (data not shown). One animal was withdrawn from the vehicle diet/saline group because it failed to gain weight. There was a decrease in food intake on day 2 in the vehicle diet/loperamide group compared to the vehicle diet/saline group $(16.88 \pm 1.17 \mathrm{~g}$ vs. $26.02 \pm 0.93 \mathrm{~g} ; \mathrm{p}<0.0001$, respectively). Moreover, the repeated measures ANOVA analysis reveals a significant difference in food intake between the experimental groups $(F=4.807 ; \mathrm{p}=0.0048)$ throughout the treatment period. Post-hoc analyses showed a decrease in food intake for vehicle diet/loperamide group compared to the vehicle diet/saline on Day $2(16.88 \pm 1.17$ g vs. $26.02 \pm 0.93 \mathrm{~g}$, respectively, $\mathrm{p}<0.0001)$ and for the pea hull diet/loperamide when compared to pea hull diet/saline at Day 2 (23.10 \pm 1.12 vs. $27.91 \pm 1.36$ g respectively; $\mathrm{p}=0.0046)$. On Days 2 and 3 there was an increase in food intake when comparing the pea hull diet/loperamide to the ve- 
hicle diet/loperamide at $\mathrm{D} 2(\mathrm{p}=0.0003)$ and $\mathrm{D} 3(33.09 \pm 2.89 \mathrm{~g}$ for the pea hull $\mathrm{diet} /$ loperamide group vs. $26.06 \pm 1.28 \mathrm{~g}$ for the vehicle diet/loperamide group; $\mathrm{p}$ $=0.0069$ ).

\subsection{Fecal Pellet Number}

As shown in Figure 2, animals in the vehicle diet/loperamide group had a decrease in the number of pellets over 24 hours ( $24.2 \pm 2.3$ pellets) compared to the vehicle diet/saline group on D1 $(47.3 \pm 3.6$ pellets; $\mathrm{p}<0.0001)$ and D2 $(47.7 \pm$ 3.6 pellets for the vehicle diet/loperamide group vs. $63.6 \pm 2.5$ pellets for the vehicle diet/saline group; $\mathrm{p}=0.002$ ).

Animals in the pea hull diet/loperamide group had a decrease in the number of pellets on Day 1 ( $35.2 \pm 2.4$ pellets) compared to the pea hull diet/saline group $(54.1 \pm 4.0$ pellets, $\mathrm{p}<0.0001)$. Similarly, on Day 2 the pea hull diet/loperamide group had a lower number of pellets compared to the pea hull diet/saline group $(48.2 \pm 2.8$ pellets vs. $75.8 \pm 4.4$ pellets, respectively; $\mathrm{p}<0.0001)$. In addition, PHF supplementation induced an increase in total fecal pellet number over $24 \mathrm{~h}$ at Day 1 in constipated rats $(35.2 \pm 2.4$ pellets for the pea hull diet/loperamide group vs. $24.2 \pm 2.3$ pellets for the vehicle diet/loperamide group; $\mathrm{p}=0.0157$ ) and at Day 2 only in non-constipated rats $(75.8 \pm 4.4$ pellets for the pea hulls/saline group vs. $63.6 \pm 2.5$ pellets for the vehicle $\operatorname{diet} /$ saline group; $\mathrm{p}=$ 0.0157).

As both interventions (PHF supplementation and loperamide injection) induced significant modification of food intake, the correlation between food intake and FPO was investigated (data not shown). The correlation factor was not significant $\left(R^{2}=0.0006\right)$, consequently the significant difference in food intake did not significantly impact neither FPO nor body weight and the significant

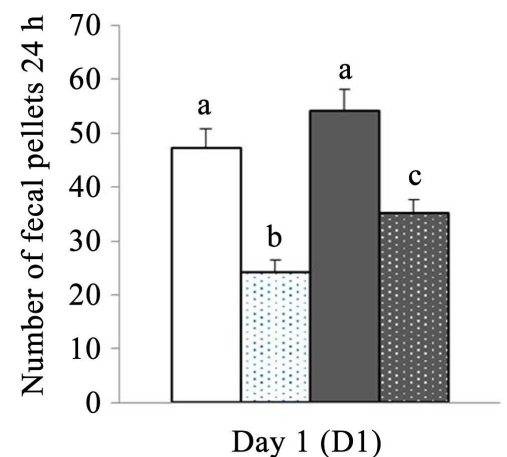

(a)

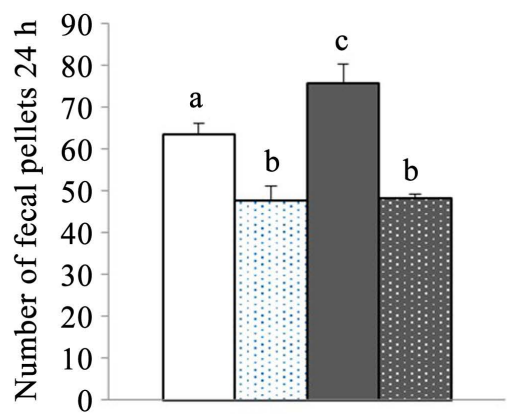

Day 2 (D2)

(b)

$\square$ Vehicle/Saline $\square$ Vehicle/Loperamide $\square$ Pea Hulls/Saline 国 Pea Hulls/Loperamide

Figure 2. Total number of fecal pellets over each $24 \mathrm{~h}$ period. (a) Total number of fecal pellets over the first $24 \mathrm{~h}$ period (D1); (b) Total number of fecal pellets over the second 24 $\mathrm{h}$ period (D2) of the vehicle/saline ( $\mathrm{n}=14$; white bars), vehicle/loperamide ( $\mathrm{n}=15$; white dotted bars), pea hulls/saline ( $n=15$; grey bars) and pea hulls/loperamide $(n=15$; grey dotted bars) groups. Data are presented as mean $+/-$ SEM. Bars sharing the same letter are not significantly different according to Fisher's PLSD Test $(\mathrm{p}<0.05)$. 
effect of PHF on FPO was due to the increase in fecal bulk linked to the consumption of highly insoluble fiber.

\subsection{Fecal Wet Weight}

As shown in Figure 3, $24 \mathrm{~h}$ fecal wet weight decreased during Day 1 in the vehicle diet/loperamide group $(1.56 \pm 0.22 \mathrm{~g})$ compared to the vehicle diet/saline group $(2.89 \pm 0.19 \mathrm{~g} ; \mathrm{p}=0.0002)$. Similarly on $\mathrm{D} 1$, the pea hull diet/loperamide group had a lower fecal wet weight than the pea hull diet/saline group on both D1 $(2.85 \pm 0.31$ g vs. $3.58 \pm 0.21 \mathrm{~g} ; \mathrm{p}=0.0322)$ and $\mathrm{D} 2(3.45 \pm 0.17 \mathrm{~g}$ vs. $4.70 \pm$ $0.24 \mathrm{~g}$, respectively; $\mathrm{p}<0.0001)$. In the non-constipated state, pea hull-treated animals showed an increase in fecal wet weight over $24 \mathrm{~h}$ when compared to the vehicle diet/saline group at Day $1(\mathrm{p}=0.0453)$ and Day $2(4.70 \pm 0.24 \mathrm{~g}$ for the pea hull/saline group vs. $3.64 \pm 0.12 \mathrm{~g}$ for the vehicle diet/saline group; $\mathrm{p}=$ 0.0007). In the constipated state, pea hull-treated animals showed an increase in fecal wet weight over $24 \mathrm{~h}$ when compared to vehicle diet/loperamide group at Day 1 only $(2.85 \pm 0.31 \mathrm{~g}$ for pea hull/loperamide group vs. $1.56 \pm 0.22 \mathrm{~g}$ for the vehicle diet/loperamide group, respectively; $\mathrm{p}=0.0003$ ).

\subsection{Fecal Dry Weight}

Fecal dry weight was lower in the vehicle diet/loperamide group compared to the vehicle diet/saline group $(0.94 \pm 0.09 \mathrm{~g}$ vs. $1.61 \pm 0.10 \mathrm{~g}$, respectively; $\mathrm{p}<0.0001)$ on Day 1 but was not different between the groups in Day 2 (Figure 4). In the pea hull diet groups, loperamide led to a decrease in fecal dry weight on Day 1 $(1.41 \pm 0.10 \mathrm{~g})$ and Day $2(2.05 \pm 0.12 \mathrm{~g})$ when compared to the saline treated group on Day $1(2.03 \pm 0.10 \mathrm{~g}, \mathrm{p}<0.0001)$ and Day $2(2.84 \pm 0.21 \mathrm{~g}, \mathrm{p}=0.0028)$. In the non-constipated state, pea hull-treated animals had increased fecal dry

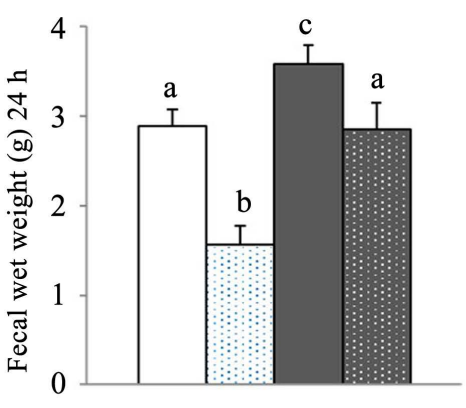

Day 1 (D1)

(a)

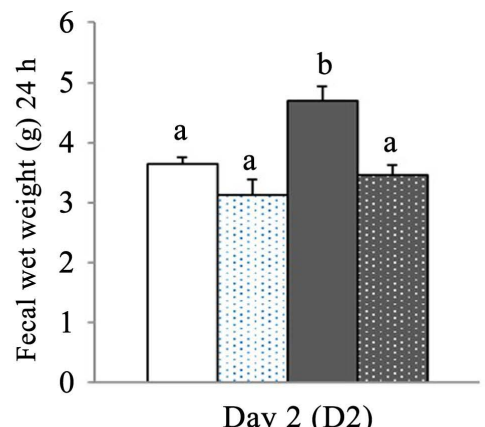

(b)

Vehicle/Saline $\square$ Vehicle/Loperamide $\square$ Pea Hulls/Saline图Pea Hulls/Loperamide

Figure 3. Fecal wet weight over each $24 \mathrm{~h}$ period. (a) Fecal wet weight over the first $24 \mathrm{~h}$ period (D1) and (b) Fecal wet weight over the second $24 \mathrm{~h}$ period (D2) of the vehicle/saline ( $\mathrm{n}=14$; white bars), vehicle/loperamide $(\mathrm{n}=15$; white dotted bars), pea hulls/saline ( $\mathrm{n}=15$; grey bars) and pea hulls/loperamide $(\mathrm{n}=15$; grey dotted bars) groups. Data are presented as mean + /- SEM. Bars sharing the same letter are not significantly different according to Fisher's PLSD Test $(\mathrm{p}<0.05)$. 


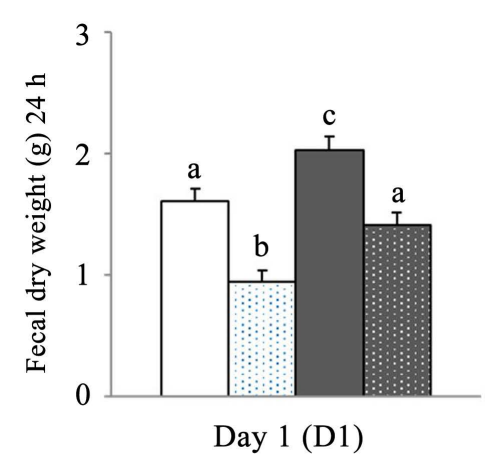

(a)

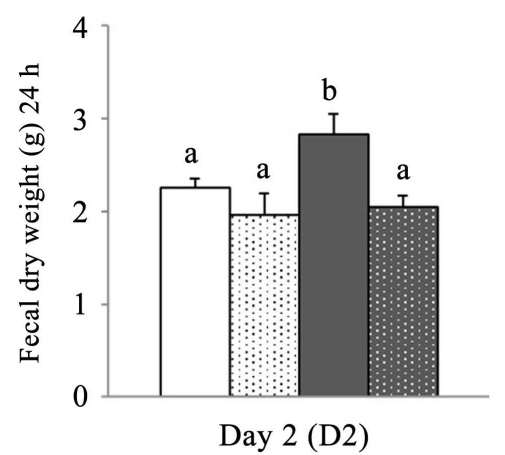

(b)

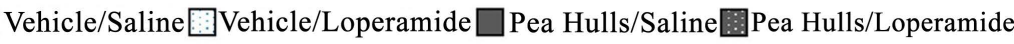

Figure 4. Fecal dry weight over each $24 \mathrm{~h}$ period. (a) Fecal dry weight over the first $24 \mathrm{~h}$ period (D1) and (b) Fecal dry weight over the second $24 \mathrm{~h}$ period (D2) of the vehicle/saline ( $\mathrm{n}=14$; white bars), vehicle/loperamide $(\mathrm{n}=15$; white dotted bars), pea hulls/saline $(n=15$; grey bars) and pea hulls/loperamide $(n=15$; grey dotted bars) groups. Data are presented as mean $+/-$ SEM. Bars sharing the same letter are not significantly different according to Fisher's PLSD Test $(\mathrm{p}<0.05)$.

weight over $24 \mathrm{~h}$ compared to vehicle diet/saline group at D1 $(\mathrm{p}=0.0044)$ and $\mathrm{D} 2(2.84 \pm 0.21 \mathrm{~g}$ for the pea hull/saline group vs. $2.26 \pm 0.09 \mathrm{~g}$ for the vehicle $\mathrm{diet} /$ saline group; $\mathrm{p}=0.0072$ ). In constipation state, pea hull-treated animals had an increase in fecal dry weight over $24 \mathrm{~h}$ when compared to vehicle diet/loperamide group at $\mathrm{D} 1$ only $(\mathrm{p}=0.0014)$.

\subsection{Cumulative FPO}

Loperamide decreased cumulative FPO over the $56 \mathrm{~h}$ experimental period in both the vehicle and pea hull diet groups (Figure 5(a)). Supplementation with pea hull fiber led to an increase in cumulative FPO over $56 \mathrm{~h}$ both in non-constipated (from the nocturnal phase of Day 1 to Day 3) and constipation states (From Day 1 to Day 3), when compared to animals fed with the vehicle diet.

These effects in total number of fecal pellets appeared sustained over $56 \mathrm{~h}$ as the same variations were observed among groups, as shown in Figure 5(c), both in non-constipated (148.07 \pm 8.48 pellets for the pea hull/saline group vs. 123.64 \pm 5.93 pellets for the vehicle diet/saline group; $\mathrm{p}=0.0077)$ and constipated conditions $(105.00 \pm 5.55$ pellets for the pea hull/loperamide group vs. $85.20 \pm 3.84$ pellets for the vehicle diet/loperamide group; $\mathrm{p}=0.0264$ ).

\section{Discussion}

Many adults in western countries consistently fail to consume the recommended daily amount of fiber, so it is not surprising that constipation and bowel irregularity are top concerns for many patients. Dietary supplementation with fiber has been shown to have a beneficial impact on constipation [5] [9]. To date, limited studies in humans with oat hull fiber and one study in PHF have suggested a positive effect of these insoluble fibers on intestinal transit. This study 


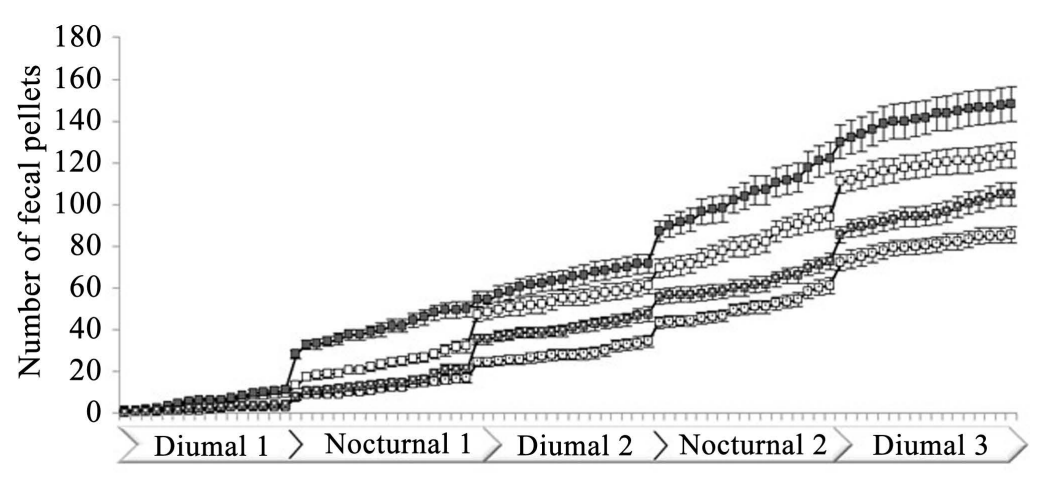

(a)

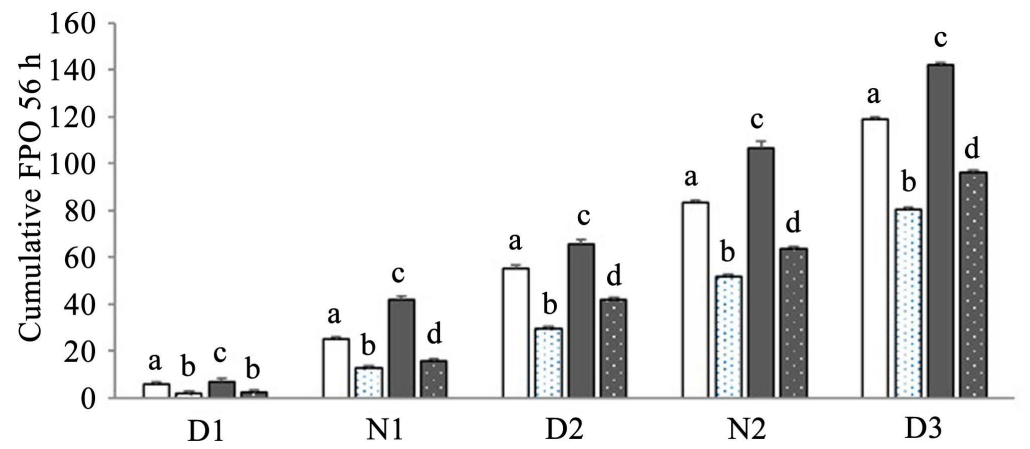

(b)

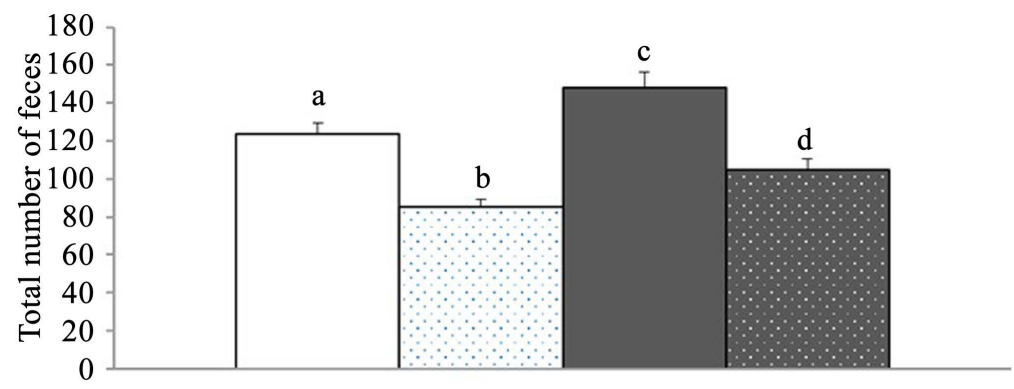

(c)

Figure 5. Cumulative FPO. (a) Total number of fecal pellets over 56 h; (b) cumulative FPO over $56 \mathrm{~h}$; and (c) total number of fecal pellets over $72 \mathrm{~h}$ (Day 1 to Day 3 ) of the vehicle/saline ( $\mathrm{n}=14$; white bars), vehicle/loperamide ( $\mathrm{n}=15$; white dotted bars), pea hulls/saline ( $\mathrm{n}=15$; grey bars) and pea hulls/loperamide ( $\mathrm{n}=15$; grey dotted bars) groups. Data are presented as mean $+/-$ SEM. Bars sharing the same letter are not significantly different according to Fisher's PLSD Test $(\mathrm{p}<0.05)$.

was designed to evaluate the effect of PHF ( $8 \%$ fiber) on fecal output in a loperamide-induced constipation rat model.

In the present study, rats were fed either a vehicle diet (4.8\% fiber) or a diet supplemented with PHF ( $8 \%$ fiber). The ground pea hulls were $93 \%$ fiber with $50 \%$ as cellulose, $25 \%$ as hemicellulose and $25 \%$ as pectin. This fiber dose was selected because it has been shown in previous studies to beneficially effect intestinal function in animal models, and it corresponds to a high fiber diet in 
humans [10] [12]. To induce constipation, rats were given an ip injection of loperamide, a $\mu$-opioid receptor agonist, used as anti-diarrheic treatment. Its mechanism is based on two different actions, decreasing intestinal peristalsis thereby slowing intestinal transit time, and by increasing water reabsorption in the intestine.

Interestingly, both wet and dry weights were impacted by supplementation, demonstrating that pea hulls had a low osmotic effect in the colon even at this dosage. The increase in FPO was not due to an increase in the water content of the feces. This means that this fiber would be well tolerated without much impact on feces consistency in humans.

Repeated loperamide injections induced rapid and sustained constipation in the treated animals, when compared to saline injections. Concerning the number of fecal pellets, loperamide stopped inducing constipation during the second experimental night and consequently lasted 36 hours although injections were still administered at 36 and $48 \mathrm{~h}$ (data not shown). The same observation can be done for the fecal wet weight whereas the fecal dry weight remains low for the loperamide groups until $48 \mathrm{~h}$ after the beginning of the experimental phase. It seems that the effect of loperamide does not last longer than $48 \mathrm{~h}$ even with repeated injections every 12 hours. In a healthy, non-diarrheic subject, loperamide effectiveness progressively decreases over time. Consequently, this model would be suitable to mimic sub-acute constipation.

Pea hulls are composed of insoluble fibers such as cellulose $(\approx 55 \%)$ and hemicellulose $(\approx 25 \%)$ and are also rich in polyphenols and soluble fibers as pectins. In our study, pea hulls were able to reverse loperamide-induced constipation as fiber supplemented groups had an increase in both FPO and total fecal pellet number compared to the constipated controls. The insoluble structure of pea hulls makes this ingredient poorly fermented in the colon. Its addition to the diet increased fecal bulk in both supplemented groups (healthy and constipated). This increased volume in the colon may have helped counter the effects of loperamide on peristalsis mediated by the $\mu$-opioid receptors. In addition to its impacts on fecal parameters, pea hull supplementation also prevented the food intake decrease linked to loperamide treatment, demonstrating no effect on appetite in the rats.

Although loperamide can only mimic a sub-acute constipation, we hypothesize that the effect of pea hulls would remain as the insoluble fibers of the hulls would be poorly fermented even after a long-term consumption [7] [17]. The positive impact of pea hulls on transit observed in the present study, is in agreement with a previous study demonstrating a decrease in transit time with a $13.5 \%$-supplementation in healthy rats [12].

\section{Conclusion}

In conclusion, in a constipation model, pea hulls were able to seriously decrease the effects of loperamide, a $\mu$-opioid receptor agonist, by increasing fecal bulk weight. Rats supplemented with PHF and treated with loperamide, had a lower 
decrease in food intake compared to the vehicle diet/loperamide-treated animals. In healthy rats, pea hulls induced higher FPO and fecal weight suggesting pea hulls may be helpful in the improvement of intestinal health.

\section{Acknowledgements}

This study was conducted by Biomeostasis (MJ, RJ \& BB) and funded by ROQUETTE (GDL \& TC). MJ performed the protocols and contribute to writing. RJ and TC designed the study and contributed to writing. BB and GDL reviewed the manuscript.

\section{Conflicts of Interest}

All the authors have declared that there is no conflict of interest.

\section{Practical Applications}

In the present study, we demonstrated that the use of the insoluble pea hull fiber could improve bowel movements not only in healthy rats but also in animals with subchronic constipation that was induced by a chemical agent.

We wanted here to demonstrate that insoluble fibers as pea hulls can have positive impacts on health even if they are poorly fermented and that this kind of food ingredient should be more largely considered to relieve moderate constipation.

\section{References}

[1] Wong, S.W. and Lubowski, D.Z. (2007) Slow-Transit Constipation: Evaluation and Treatment. ANZ Journal of Surgery, 77, 320-328. https://doi.org/10.1111/j.1445-2197.2007.04051.x

[2] Mugie, S.M., Benninga, M.A. and Di, L.C. (2011) Epidemiology of Constipation in Children and Adults: A Systematic Review. Best Practice \& Research: Clinical Gastroenterology, 25, 3-18. https://doi.org/10.1016/j.bpg.2010.12.010

[3] Buttriss, J.L. and Benelam, B. (2010) Nutrition and Health Claims: The Role of Food Composition Data. European Journal of Clinical Nutrition, 64, S8-S13. https://doi.org/10.1038/ejcn.2010.203

[4] Grundy, M.M., Fardet, A., Tosh, S.M., Rich, G.T. and Wilde, P.J. (2018) Processing of Oat: The Impact on Oat's Cholesterol Lowering Effect. Food \& Function, 9, 1328 1343. https://doi.org/10.1039/C7FO02006F

[5] Christodoulides, S., Dimidi, E., Fragkos, K.C., Farmer, A.D., Whelan, K. and Scott, S.M. (2016) Systematic Review with Meta-Analysis: Effect of Fibre Supplementation on Chronic Idiopathic Constipation in Adults. Alimentary Pharmacology \& Therapeutics, 44, 103-116. https://doi.org/10.1111/apt.13662

[6] Rao, S.S., Yu, S. and Fedewa, A. (2015) Systematic Review: Dietary Fibre and FODMAP-Restricted Diet in the Management of Constipation and Irritable Bowel Syndrome. Alimentary Pharmacology \& Therapeutics, 41, 1256-1270. https://doi.org/10.1111/apt.13167

[7] Suares, N.C. and Ford, A.C. (2011) Prevalence of, and Risk Factors for, Chronic Idiopathic Constipation in the Community: Systematic Review and Meta-Analysis. The American Journal of Gastroenterology, 106, 1582-1591. 
https://doi.org/10.1038/ajg.2011.164

[8] McRorie, J.W. (2015) Evidence-Based Approach to Fiber Supplements and Clinically Meaningful Health Benefits, Part 1: What to Look for and How to Recommend an Effective Fiber Therapy. Nutrition Today, 50, 82-89.

https://doi.org/10.1097/NT.0000000000000082

[9] Axelrod, C.H. and Saps, M. (2018) The Role of Fiber in the Treatment of Functional Gastrointestinal Disorders in Children. Nutrients, 10, 1650.

https://doi.org/10.3390/nu10111650

[10] Dahl, W.J., Whiting, S.J., Healey, A., Zello, G.A. and Hildebrandt, S.L. (2003) Increased Stool Frequency Occurs When Finely Processed Pea Hull Fiber Is Added to Usual Foods Consumed by Elderly Residents in Long-Term Care. Journal of the American Dietetic Association, 103, 1199-1202.

https://doi.org/10.1016/S0002-8223(03)00977-5

[11] Stephen, A.M., Champ, M.M., Cloran, S.J., et al. (2017) Dietary Fibre in Europe: Current State of Knowledge on Definitions, Sources, Recommendations, Intakes and Relationships to Health. Nutrition Research Reviews, 30, 149-190. https://doi.org/10.1017/S095442241700004X

[12] Hansen, I., Knudsen, K.E. and Eggum, B.O. (1992) Gastrointestinal Implications in the Rat of Wheat Bran, Oat Bran and Pea Fibre. British Journal of Nutrition, 68, 451-462. https://doi.org/10.1079/BJN19920103

[13] Kim, J.E., Lee, Y.J., Kwak, M.H., et al. (2014) Metabolomics Approach to Serum Biomarker for Loperamide-Induced Constipation in SD Rats. Laboratory Animal Research, 30, 35-43. https://doi.org/10.5625/lar.2014.30.1.35

[14] Kim, J.E., Go, J., Koh, E.K., et al. (2016) Gallotannin-Enriched Extract Isolated from Galla Rhois May Be a Functional Candidate with Laxative Effects for Treatment of Loperamide-Induced Constipation of SD Rats. PLOS ONE, 11, e0161144. https://doi.org/10.1371/journal.pone.0161144

[15] Sabiu, S. and Ashafa, O.T. (2016) Toxicological Implications and Laxative Potential of Ethanol Root Extract of Morella serrata in Loperamide-Induced Constipated Wistar Rats. Pharmaceutical Biology, 54, 2901-2908. https://doi.org/10.1080/13880209.2016.1193885

[16] Wintola, O.A., Sunmonu, T.O. and Afolayan, A.J. (2010) The Effect of Aloe Ferox Mill. in the Treatment of Loperamide-Induced Constipation in Wistar Rats. BMC Gastroenterology, 10, 95. https://doi.org/10.1186/1471-230X-10-95

[17] Cook, I.J., Talley, N.J., Benninga, M.A., Rao, S.S. and Scott, S.M. (2009) Chronic Constipation: Overview and Challenges. Neurogastroenterology \& Motility, 21, 1-8. https://doi.org/10.1111/j.1365-2982.2009.01399.x 